\title{
Die Rolle des Raumes als zu erklärender und als erklärender Faktor Zur Klärung einer methodologischen Grundrelation in der Geographie
}

\section{Der Anstoß zu diesem Beitrag}

Den letzten Anstoß, dieses länger schon verfolgte und bisher nur randlich mitbehandelte Thema (vgl. z. B. КÖСK 1987.1; 1987.2; 1991) zum Gegenstand einer eigenständigen Erörterung zu machen, gab WERLENS «Sozialgeographie alltäglicher Regionalisierungen» (Bd. 1, 1995). Vor dem Hintergrund des Einwandes, «daß nur materielle Gegebenheiten eine erdräumlich lokalisierbare Existenz aufweisen und dementsprechend auch raumwissenschaftlich untersucht werden können, nicht aber (immaterielle) subjektive Bewußtseinsgehalte, soziale Normen und kulturelle Werte» (S. 3), sieht WERLEN ein doppeltes Dilemma: Das eine folgt aus seinem Ziel, als Kern seiner Sozialgeographie «das alltägliche Geographie-Machen wissenschaftlich zu erforschen» (S. 236). Da dies wesentlich individuelle wie kollektive Bewußtseinserforschung bedeute, Bewußtseinsinhalte sich jedoch dem räumlichen Paradigma entzögen (siehe oben), wäre dies keine Geographie mehr - jedenfalls soweit diese räumlich definiert ist. Das andere ergibt sich aus WERLENS Behauptung, der moderne relationale und als solcher rein formale, konstruktivistische Raumbegriff entziehe sich der empirischen wissenschaftlichen Erforschung (S. 4, 6, 241). Daher ist nach WERLEN «in der Kultur- und Gesellschaftsforschung die raumzentrierte Suche nach dem Kulturellen und Sozialen durch die Klärung der räumlichen Bedingungen für die Konstitution der Gesellschaften und Kulturen zu ersetzen ... Es geht also um den Perspektivenwechsel von der sozial-orientierten Raumforschung zur geographischen Gesellschaftsforschung» (S. 4). Im AV/UV-Kontext ausgedrückt, meint WERLEN also: Man tausche die bisherige AV (Abhängige Variable) der (Sozial-)Geographie, den Raum, gegen die Gesellschaft/Kultur als der anthropogeographisch bisher wichtigsten UV (Unabhängigen Variablen), mache also die bisherige UV zur AV und betrachte den Raum fortan als gesellschaftlich erklärungsstarke UV. «So stehen nicht 〈Raum», die bloße 〈Geographie〉 der Dinge oder die Suche nach Gesetzmäßigkeiten in deren Anordnung, im Zentrum, sondern vielmehr jene Handlungen der Subjekte, über welche deren «Geographien» hergestellt und reproduziert werden» (S. 5).

Es soll nun nicht behauptet oder näher untersucht werden, daß oder inwieweit es sich hierbei um eine rein zweckrationale, um nicht zu sagen utilitaristische Argumentation bzw. Konstruktion WERLENS zur «Rettung» seiner "Sozialgeographie» als «noch geographisch» handelt. Immerhin müßte man, folgt man WERLENS ja länger schon favorisiertem Vorschlag, die paradigmatische Logik zumindest der Anthropogeographie geradewegs auf den Kopf stellen. Auch soll hier nicht die von WERLEN behauptete angebliche Notwendigkeit dieser «Modernisierung», dieses Paradigmenwechsels (S. 5, 6) erörtert werden. Nur weil WERLEN das klassische geographische Paradigma nicht paßt bzw. mit der von $\mathrm{ihm}$ anvisierten Sozialgeographie nicht verträglich ist, muß ja nicht gleich eine ganze Disziplin ihr Paradigma wechseln. Es könnte sich ja WERLEN eine paradigmatisch genehme(re) Disziplin suchen. Nein: WERLENS Konstruktion dient hier lediglich als eben letzter Anstoß, die Rolle des AV/UV-Konzeptes in der Geographie einmal explizit, wenngleich nur in Aufsatzform, zu erörtern. Allerdings ist nicht auszuschließen, daß sich als Folge davon auch WERLENS Dilemmata verflüchtigen.

\section{Methodologische Ausgangspositionen}

Grundlage der nachfolgenden Ausführungen ist das relationale, und zwar chorologische Raumverständnis. Implizit oder explizit bildet es nach wie vor die weithin anerkannte «methodologische Mitte» der Geographie. Ein methodologisch ernsthafter Grund, davon abzurücken, ist nicht zu sehen. Und ob es, auch mit Blick auf die Außenwirkung, fachpolitisch klug ist, diesen «Ankerpunkt» ungeachtet fehlender plausibler Notwendigkeit (wieder einmal) in Frage zu stellen, ist eine eigene, hier nicht näher zu verfolgende Frage.

Ohne ihn jedenfalls könnte sich die Geographie kaum, wenn überhaupt, legitimieren. Da hilft auch das Realobjekt «Geosphäre» nicht. Zwar ist diese als integrative Wechselwirkungsgesamtheit keiner sonstigen Disziplin Gegenstand. Die Einzelsphären wie auch verschiedene Einzelbereiche aus ihr werden jedoch immer schon auch von anderen Wissenschaften, wenngleich unter anderen

Helmuth Köck, Prof. Dr. Dr., Universität Koblenz-Landau, Abt. Landau, Im Fort 7, D-76829 Landau 
Fragestellungen, untersucht. Die disziplinäre Behauptung und Rechtfertigung diesen gegenüber ist der Geographie dann nur durch ihre Fragestellung, eben die räumliche bzw. chorologische Weltperspektive, mit der sie die Geosphäre betrachtet, möglich. Erst durch die Verknüpfung des chorologischen Erkenntnisobjektes mit dem geosphärischen Realobjekt bzw. beliebigen Teilen von ihr als geographiespezifischer - und nur geographiespezifischer - AV (dazu später!) gelingt der Geographie auch weiterhin die begründete Positionierung im Reigen der Geo- und Raumwissenschaften.

$\mathrm{Da} \beta$ der Raum als geographisches Erkenntnisobjekt dabei formal-relational statt gegenständlich-absolut definiert ist, ist geographieintern offenbar unstrittig. Allenfalls die dimensionsstufenspezifische Geltung des chorologischen Paradigmas ist nicht hinreichend geklärt. Denn seit NEEF 1963, neben bzw. zwischen der topologischen sowie der geosphärischen, eine eigenständige chorologische Dimensionsstufe vorgeschlagen und das chorologische Paradigma nur dieser Dimensionsstufe zugeordnet hat, wird die chorologische Betrachtung, allenfalls geringfügig modifiziert, unverständlicherweise in der Regel eben nur dieser Betrachtungsstufe zugewiesen. Dabei macht der Begriff «chorologisch» keinerlei Größen-, sondern nur Relations- oder Strukturaussagen. Raumrelationale, also chorologische Strukturen gibt es jedoch auf jeder beliebigen Größenstufe, und nicht nur auf einer bestimmten. Überdies widerspricht NEEF sich mit seinem Vorschlag selbst. Denn gemäss seiner axiomatischen Grundlegung von 1956 (und später 1967) sind alle geographischen Erscheinungen durch eine räumliche Ordnung, mithin chorologisch gekennzeichnet. Folglich gilt dies unabhängig von der Größenstufe der jeweiligen geographischen Erscheinungen, und dies nicht nur im Sinne einer axiomatischen Setzung, sondern auch und vor allem vor dem Hintergrund empirischer Evidenz. Ohne auf diesen logischen Bruch hier näher eingehen zu können, gilt im folgenden jedenfalls: Das chorologische Paradigma gilt für alle Größenstufen geographischer Weltbetrachtung. Was es von Größenstufe zu Größenstufe unterscheidet, ist einzig sein Generalisierungsgrad.

Nun kann der Raum methodologisch bekanntlich zwei, gegebenenfalls sogar drei verschiedene Rollen spielen, und zwar - in der Terminologie von MAYNTZ/HOLM/ HÜBNER $\left({ }^{2} 1971\right.$, S. 30$)$ und (ergänzend) ATTESLANDER ( ${ }^{7} 1993$, S. 204/205) - die Rolle einer

- abhängigen, zu erklärenden Variablen (AV = Explanandum),

- unabhängigen, erklärenden Variablen (UV = Explanans),

- intervenierenden, «sich in den Zusammenhang zwischen einer abhängigen und unabhängigen beiläufig einmischende[n] Variable[n]» (ATTESLANDER ${ }^{7} 1993$, S. 205) (IV = Randbedingung).

Die klassische Rolle des Raumes ist sicher die einer AV bzw. des Explanandums. Diese ist beispielsweise dann gegeben, wenn es darum geht, die Reihung von Inseln oder die innerstädtische Polyzentrizität zu erklären.
Doch auch die Rolle des Raumes als UV, als selbst erklärungsmächtiger Faktor also, gehört zum Standard. Dieser Fall liegt beispielsweise dann vor, wenn zur Erklärung von Burgenstandorten auch räumliche Lagebeziehungen berücksichtigt werden. Ungewohnter ist demgegenüber die Rolle des Raumes als IV. Ein Beispiel hierfür liegt etwa dann vor, wenn es das Vorkommen von Wüstenstaub aus der Sahara in Deutschland zu erklären gilt und dabei auch die zwar nicht unmittelbar verursachenden, wohl aber vermittelnden, steuernden Einflüsse zu berücksichtigen sind, die von dem zwischen Quellund Zielgebiet liegenden Raum mit seinen spezifischen, vor allem physischgeographischen Strukturen ausgehen. Es stellt sich allerdings die Frage, ob man die IV statt als eigenständigen Variablentyp nicht eher als Bestandteil des Explanans und somit als eine spezielle UV-Art betrachten muß. Denn genaugenommen stellen sie ja Randbedingungen dar, und die gehören seit HEMPEL/ OPPENHEIM (1948) zum klassischen Bestand des Explanans, also der UV. Entsprechend soll auch hier fortan nur noch von AV und UV die Rede sein.

Folgt man nun dem Vorschlag weINGARTNERS $\left({ }^{2} 1978\right.$, S. 130), Wissenschaften nach der Form derjenigen Klasse von Sätzen einzuteilen, die in den betreffenden Wissenschaften erklärt bzw. begründet werden, dann erhalten die AV, und so hier der Raum als AV, eine methodologisch herausragende Position. Sie werden «zum entscheidenden Sammelpunkt einer Fachdisziplin; sie sichern deren inhaltlichen Zusammenhang. Sie machen den Relevanzfilter einer Disziplin aus. Sie sind sozusagen die Brille, durch die (ein Fach) die Sachverhalte der Welt betrachtet» (DÜRR 1979, S. 18, jedoch ohne Bezug auf WEINGARTNER).

Diese methodologische Sonderstellung der AV läßt sich plausibel begründen. Drei Argumente seien genannt:

- Das einleuchtendste Argument läßt sich verkürzt auf die epistemische Formel «ohne AV keine UV» bringen. Das heißt: Im Unterschied zur Realität, in der es ohne UV keine AV gibt, bilden im Erkenntnisprozeß zu erklärende oder zu begründende Sätze als Aussagen über Seiendes oder Seinsollendes den Ausgangspunkt, von dem aus sich überhaupt erst die Frage nach dem Warum, also nach der Erklärung (von Seiendem) oder Begründung (von Seinsollendem) stellt. Umgekehrt repräsentieren die AV den Gegenstandsbereich, mit dem sich eine Wissenschaft beschäftigt und dessen Kausalität (UV) sie aufdecken will.

- Die erklärenden Sätze, also die UV, gewinnt eine jede Wissenschaft nicht nur aus dem je eigenen «epistemischen Korpus» (KUTSCHERA 1972, I, S. 228, 246), sondern auch aus demjenigen anderer Wissenschaften (Beispiel: Erklärung der globalen räumlichen Ordnung der Hochgebirge unter Hinzuziehung unter anderem geologischer, geotektonischer, geochemischer usw. Erkenntnisse). Mithin ist die Zuordnung der im Explanans verwendeten Sätze zu einer und nur einer - Wissenschaft und folglich die Identi- 
fikation einer Wissenschaft anhand des Explanans nicht möglich.

- Schließlich verwendet man in der Erklärung nicht nur disziplinär, sondern vielfach auch methodologisch unterschiedliche Aussagen, so etwa empirische zusammen mit normativen oder Wertaussagen, so daß auch aus dieser Sicht eine Zuordnung der Aussagen zu einem bestimmten Wissenschaftstyp geschweige denn einer bestimmten Einzelwissenschaft nicht möglich ist.

Aus diesen Gründen gilt hier also WEINGARTNERS Vorschlag als dritte und entscheidende methodologische Ausgangsposition. Zwar ließe sich noch einwenden, WEINGARTNER spreche ja von der (methodologischen) «Form» und nicht vom (paradigmatischen oder objekttheoretischen) «Gehalt» der zu erklärenden bzw. zu begründenden Sätze als Einteilungskriterium, so daß es eher um die Unterscheidung etwa zwischen empirischen, deskriptiven, normativen, formalen usw. denn zwischen beispielsweise Raum-, Sozial-, Natur-, Geisteswissenschaften o. ä. gehe. Doch macht es methodologisch keinen Unterschied, außer der «Form» auch den «Gehalt» der AV zum Einteilungskriterium zu machen. Dafür sprechen unter anderem folgende Argumente:

- Nicht die «Form» oder der «Gehalt» eines Satzes ist bei WEINGARTNER letztlich essentiell, sondern dessen methodologischer Status als AV. Setzt man nun alternativ zur Form den Gehalt ein, so ändert dies nichts an WEINGARTNERS Vorschlag, die AV und nicht die UV als Einteilungskriterium zugrunde zu legen.

- Die eigentliche und substantiell gehaltvolle Differenzierung und Systematisierung von Wissenschaften ist ja paradigmatisch-objekttheoretischer und nicht formaler Art. Während letztere lediglich zu Wissenschaftstypen wie etwa empirischen, normativen oder ähnlichen führt, erlaubt erstere eine Einteilung in Gruppen von Wissenschaften bzw. dann Einzelwissenschaften, die mit konkreten Gegenstandsklassen oder Betrachtungsweisen der realen Welt korrespondieren.

- Bereits die weiter oben entfaltete Begründung der Plausibilität der Position weINGARTNERS legt dar, daß es in einzelwissenschaftlichen Erkenntnisprozessen ja gerade auf den Gehalt und weniger auf die Form der Aussagen ankommt. Speziell das zweite dort genannte Argument zwingt geradezu dazu, auch und vor allem den Gehalt der AV als Einteilungskriterium zu verwenden.

Damit diese somit begründete Ausgangsposition gar nicht erst mißverstanden werden kann, sei ergänzend noch einmal DÜRR zitiert. Als plausible Konvention schlägt er vor (1979, S. 19): «Unnachgiebigkeit in der Orientierung der Forschungsarbeit auf die AV des Faches, größtmögliche Flexibilität in der Auswahl der UV». Spricht man statt von "Flexibilität» eher von «Offenheit» in der Auswahl der UV, so macht diese Formel deutlich, daß es für die Suche nach erklärungsbedeutsamen UV keinerlei und somit auch keine disziplinären Grenzen gibt, daß jene also außer in der eigenen Disziplin überall gesucht werden können bzw. letztlich müssen (vgl. auch DACEY 1979, S. 41; WEINGARTNER ${ }^{2} 1978$, S. 130, Fn. 1). Wenn BAHRENBERG (1987.1, S. 143) dem Verfasser diesbezüglich dagegen vorhält, «demnach müßte ja das Explanandum der einen Disziplin das Explanans einer anderen sein und umgekehrt», so bleibt darauf nur zu sagen: Genau so ist es, wenngleich in der Regel nur partiell, d. h. nur einzelne UV des jeweiligen Explanans bzw. AV des jeweiligen Explanandums betreffend (vgl. auch DÜRR 1979, S. 17/18). Und BAHRENBERGS (S. 143) weitergehende Befürchtung, auf diese Weise könne eine Wissenschaft «wahrscheinlich überhaupt nicht funktionieren», wird nicht nur durch die trotz allem und gerade deshalb funktionierende wissenschaftliche Praxis widerlegt, sondern auch durch methodologische Argumente selbst: Denn wäre es nicht so, dürfte es überhaupt nur eine allzuständige Wissenschaft geben, die sowohl die Beschreibung als auch die Erklärung sämtlicher denkbaren Sachverhalte leistet. Ebenso unsinnig wie dieser Gedanke wäre dessen Alternative, daß nämlich alle Wissenschaften gleichermaßen allzuständig wären. Die aus erkenntnistheoretischen wie auch erkenntnispraktischen Gründen unabweisbare Parzellierung des komplex vernetzten empirischen Forschungsobjektes Welt bzw. die jener entsprechende Spezialisierung in je teilzuständige Einzelwissenschaften bringt gewissermaßen automatisch den - heute würde man sagen - «transdisziplinären» AV/UVAustausch mit sich. Daß andererseits dieser formalrelational-konstruktivistische Raum laut WERLEN (vgl. Kap. 1) nicht empirisch-wissenschaftlich erforscht werden könne, ist durch nichts zu begründen. Sämtliche einschlägigen Kriterien empirisch-wissenschaftlichen Erkenntnisstrebens (terminologische Normierung, methodische Normierung, intersubjektive Überprüfbarkeit bzw. Kritisierbarkeit) lassen sich strikt erfüllen (womit nicht gesagt ist, daß sie immer erfüllt werden). Zumindest glaubwürdig, wenngleich gleichwohl unbegründet, wäre diese Behauptung WERLENS, wenn er sie in gleichem Atemzug auf die von ihm wissenschaftlich verfolgten Konstrukte «Gesellschaft» und «Kultur» bezöge wie erst recht natürlich auf die von ihm angestrebte Erforschung des «Bewußtseins».

\section{Ziel}

Vor dem Hintergrund dieser Problem- und Ausgangslage ist es nun das Ziel dieses Beitrages, das methodologische Potential des «Raumes als $A V$ » quer durch die wichtigsten innergeographischen methodologischen Fragenkreise auszuloten.

\section{Das methodologische Potential des Raumes als AV}

\subsection{Definition und Abgrenzung der Geographie}

Die Rolle des Raumes als AV zeigt sich schon bei der immer wieder auftretenden Notwendigkeit, die Geogra- 
phie zu definieren und gegenüber anderen Wissenschaften abzugrenzen (jedenfalls methodologisch, wenngleich weniger in der wissenschaftlichen Praxis). Gewiß läßt sich im wissenschaftlichen Alltag auch weiterhin gut mit Formeln arbeiten wie etwa

«Die Geographie ist die Wissenschaft

- von der räumlichen Ordnung der Erde bzw. Erdoberfläche,

- von der geosphärischen räumlichen Ordnung,

- von der chorologischen Struktur der Erde bzw. Erdoberfläche

- von den räumlichen Strukturen der Erde bzw. Erdoberfläche,

$-\ldots . »$

Methodologischen Ansprüchen genügen sie jedoch nicht vollends. Zwar sagen sie aus, was Gegenstand und Fragestellung der Geographie sind, und enthalten insofern die beiden notwendigen Grundinformationen. Methodologisch hinreichend sind sie jedoch gleichwohl nicht, da sie keine eindeutige Abgrenzung zu anderen Wissenschaften ermöglichen; denn Aussagen über die räumliche Ordnung der Erde bzw. Erdoberfläche können auch in anderen Wissenschaften vorkommen bzw. kommen auch in anderen Wissenschaften vor.

Unter Aufnahme des AV-Kriteriums in das Definiens ist dies jedoch möglich. Versteht man unter einer Wissenschaft mit ALBERT (1987, S. 18) ein System von «Aussagen» (statt, wie WEINGARTNER [ ${ }^{2} 1978$, S. 53, 130], ein System von «Sätzen»; vgl. hierzu KöCK 1991, S. 43/44), so ist die Geographie dann dasjenige System von Aussagen, dessen AV chorologische geosphärische Sachverhalte beinhalten. Mithin ist die Geographie, entgegen BAHRENBERGS (1987.2, S. 225/226) Unmöglichkeitsbehauptung, eine im Substrat der Geosphäre verankerte chorologische bzw. Raumwissenschaft (vgl. auch DÜRR 1979, S. 59, 60 sowie entsprechende Belege in KÖCK 1987.1, S. 190/191). Entsprechend ist ein spezielles Aussagensystem dann - und nur dann - geographisch, wenn seine abhängigen Variablen einen chorologischen geosphärischen Sachverhalt zum Gegenstand haben.

Besteht ein Aussagensystem nur aus AV, so kann seine Geographiespezifik unmittelbar geprüft werden. Ein Beispiel hierzu wäre etwa: «Die glazialen Ablagerungen im Norddeutschen Tiefland sind zonal geordnet bzw. bilden eine glaziale Serie. Von der Ostseeküste landeinwärts folgen die Grundmoränen-, die Endmoränen-, die Sander- und die Urstromtalzone aufeinander. Die Grundmoränenzone verläuft ... und besteht aus ... Die Endmoränenzone verläuft ... und besteht aus ... usw.» Zwar könnten diese deskriptiven Aussagen auch erklärende Funktion haben, etwa in bezug auf den entsprechenden zonalen Wandel der agrarischen Nutzung oder der ursprünglichen natürlichen Vegetation. Doch da ein derartiger Kontext hier nicht gegeben oder jedenfalls nicht angenommen ist, da dieses Aussagensystem vielmehr nur die chorologische Beschaffenheit des geosphärischen Systems «glaziale Serie» zum Gegenstand hat, ist sein deskriptiver, also AV-Charakter evident. Ebenso evident ist sein geosphärisch-chorologischer
Gehalt (landeinwärtige zonale/seriale räumliche Ordnung der glazialen Sediment- und Formentypen). Mithin handelt es sich um ein geographisches Aussagensystem im Sinne des WEINGARTNERschen Kriteriums. Weniger evident ist die Geographiespezifik eines Aussagensystems, wenn dieses eine Theorie, also ein System von unabhängigen Variablen bzw. ein Explanans darstellt. In diesem Fall wäre zunächst zu prüfen, welches die AV sind, die durch die betreffende Theorie erklärt werden, und welchem Paradigma sie "gehorchen». Denn aufgrund des oben Gesagten ist eine Theorie «dann und nur dann geographisch, wenn sie auf die Erklärung von räumlicher Differenziertheit ... zielt, d. h. ... eine der konkreten geographischen Grundkategorien als abhängige Variable wählt» (DÜRR 1979, S. 18). Dabei spielt es keine Rolle, welcher Art und Herkunft ihre unabhängigen Variablen sind (vgl. auch WEINGARTNER ${ }^{2} 1978$, S. 130, Fn. 1; DACEY 1979, S. 41; KÖCK 1987.1, S. 189). Ist das betreffende Explanandum dann aber konsistent zum chorologischen Paradigma, so handelt es sich um eine geographische Theorie, auch wenn deren UV ganz oder teilweise anderen Disziplinen entnommen sind.

Nehmen wir an, es handle sich um eine Theorie zur Erklärung der oben erwähnten "glazialen Serie», allgemein oder angewandt z. B. auf den norddeutschen Fall. $\mathrm{Da}$ die geochorologische Spezifik der betreffenden AV evident ist (siehe oben), ist die Theorie folglich eine geographische Theorie, auch wenn sie außer geographischen UV auch solche etwa aus der Physik (z. B. Gravitation, Energie) oder der Glaziologie (z. B. Bildung und Verhalten von Inlandeis) benutzt. Desgleichen handelt es sich bei der Erklärung etwa der räumlichen Verbreitung von Windenergierotoren um eine geographische Theorie, auch wenn hierbei neben geographiespezifischen UV auch solche der Politologie (Energieeinspeisungsgesetz, Umweltschutz, Ressourcenschonung ...), der Ökonomie (z. B. Gewinnstreben) oder der Bauphysik (z.B. bauliche Standorteignung) Verwendung finden; denn auch in diesem Fall genügt das Explanandum ja dem geochorologischen Paradigma. Wie verhält es sich demgegenüber mit Theorien zur Erklärung der räumlichen Verbreitung beispielsweise von Religionen, Mundarten oder Kulturen (vgl. zum letzteren auch WERLEN). Wenngleich in allen drei Fällen auch geographische UV zu berücksichtigen sind (z. B. Reliefstruktur, Entfernungen, klimatische Bedingungen, Verkehrseignung...), so handelt es sich gleichwohl nicht um geographische Theorien, da die $\mathrm{AV}$ in allen drei Fällen keine geosphärischen, wenngleich räumlich codierbare Sachverhalte sind. Oder: Eine Theorie zur Erklärung der Ausdehnungskoeffizienten der Mineralbestandteile etwa von Granit ist gleichfalls keine geographische Theorie, auch wenn Granit Bestandteil der Geosphäre und Ausdehnung ein räumliches Phänomen ist; denn hierbei geht es um die Erklärung rein physikalischer Materialeigenschaften, nicht dagegen um geosphärische räumliche Strukturen. Und soweit es dabei eben auch um den Raum geht, handelt es sich um den gegenständli- 
chen, absoluten und nicht um den relationalen, relativen Raum, überdies fast schon mikroskopischer Größenordnung.

Vor diesem Hintergrund wird ersichtlich, daß auch das eingangs zitierte Forschungsparadigma WERLENS kein geographisches Paradigma darstellt. Denn zwar ist es konsistent zur geographischen Theoriebildung und zudem unabweisbar, auch Entscheidungs- und Handlungsmotive als Ursachen räumlicher Strukturen aufzudecken (z. B.: Was waren nun wirklich die Motive für die Anlage des fächerförmigen Straßengrundrisses im frühen Karlsruhe?). Nichtgeographisch ist es jedoch, wenn WERLEN «die Klärung der räumlichen Bedingungen für die Konstitution der Gesellschaften und Kulturen» anstrebt. Zwar stellen die im Rahmen dieser Theoriebildung gesuchten räumlichen Bedingungen für sich betrachtet abhängige Variablen, je nach Kontext gegebenenfalls auch unabhängige Variablen der Geographie dar. Da sie in WERLENS Kontext jedoch der Erklärung eindeutig nichtgeographischer AV, nämlich der «Konstitution der Gesellschaften und Kulturen», dienen sollen, kann die betreffende angezielte Theorie gemäß obigen Kriterien keine geographische Theorie sein. Folglich kann auch das gesamte Forschungsparadigma kein geographiebegründendes Paradigma sein; vielmehr müßte man es wohl eher als politikwissenschaftlich, sozialwissenschaftlich, ethnologisch o. ä. bezeichnen.

An diesem Beispiel wird zugleich der methodologische Statuswechsel erkennbar, den dieselbe Aussage erfahren kann, nämlich den zwischen bald AV, bald UV. Weiterhin wird hieran die prinzipielle transdisziplinäre Verwendungsmöglichkeit derselben Aussage erkennbar, wobei dies mit einem methodologischen Statuswechsel verbunden sein kann (siehe oben), jedoch auch methodologische Statusgleichheit bedeuten kann. So könnte beispielsweise das sogenannte «Einspeisungsgesetz» in bezug auf die Geographie (Erklärung von Windenergierotorstandorten), die Betriebswirtschaftslehre (Erklärung des privaten Baus und Betriebs von Windenergieanlagen) oder die Energiewissenschaft (Erklärung der Verschiebung der Anteile der verschiedenen Energieträger) die Rolle einer UV, in bezug auf die Politik- oder Rechtswissenschaft (Erklärung/Begründung seiner Notwendigkeit, Rechtmäßigkeit, Legitimität) dagegen diejenige einer AV spielen.

\subsection{Innerdisziplinäre Systematik der Geographie}

Nun ist WEINGARTNERS in 4.1 auf die Geographie angewandtes Kriterium zur Einteilung von Wissenschaften natürlich für den interdisziplinären Gebrauch gedacht. Doch ist die Frage der innerdisziplinären Untergliederung logisch äquivalent derjenigen im interdisziplinären Zusammenhang. Ein Unterschied besteht lediglich im begriffs- bzw. systemhierarchischen Niveau, auf dem das Kriterium zur Anwendung kommt.

Wendet man nun WEINGARTNERS Kriterium auf die innere Untergliederung der Geographie an, so ergibt sich:
Teildisziplinen der Geographie sind Aussagensysteme, deren AV bestimmte Klassen chorologischer geosphärischer Sachverhalte beinhalten, oder: Die AV von Aussagensystemen über bestimmte Klassen chorologischer geosphärischer Sachverhalte definieren Teildisziplinen der Geographie. Konkretisiert heißt dies dann zum Beispiel: Geomorphologie ist dasjenige Aussagensystem, dessen AV chorologische geomorphologische Sachverhalte der Geosphäre beinhalten. Oder: Stadtgeographie ist dasjenige Aussagensystem, dessen AV chorologische städtische Sachverhalte der Geosphäre beinhalten. Welcher innergeographischen Art und Herkunft die erklärenden Aussagen dabei sind, zum Beispiel fluviatiler (hydrographischer) Art und Herkunft im Falle der Geomorphologie (vgl. etwa Rheingraben) oder zum Beispiel geomorphologischer Art und Herkunft im Falle der Stadtgeographie (vgl. etwa Schwemmfächer als Siedlungsstandorte), spielt definitionsgemäß keine Rolle. Entscheidend ist, daß die jeweiligen AV Klassen chorologischer geosphärischer Sachverhalte beinhalten.

Soweit zur Erklärung der betreffenden AV auch nichtgeographische UV Verwendung finden (zum Beispiel geotektonischer Art und Herkunft im Falle des Rheingrabens, historischer Art und Herkunft im Falle von Siedlungsstandorten), sind diese dann zwar Bestandteile bestimmter geographischer Theorien (da sie ja der Erklärung geographischer AV dienen werden) und damit des geographischen Aussagensystems; für sich betrachtet, herausgelöst also aus dem geographischen Erklärungszusammenhang, sind sie jedoch wiederum dem Repertoire der jeweiligen Herkunftsdisziplinen zuzurechnen, tragen sie also nicht zur Konstituierung einer geographischen Teildisziplin bei.

$\mathrm{Ob}$ ein gegebenes Arbeitsgebiet nun den Status einer geographischen Teildisziplin hat oder nicht, läßt sich anhand des hier benutzten Kriteriums prüfen. Bei den eher klassischen Gebieten, wie sie in den einschlägigen Systematisierungen der Geographie etwa durch BOBEK (1957, S. 143), UHLIG (1970, S. 28) oder LESER (1980, S. 68/69) ausgewiesen sind, ist dies offenkundig der Fall: Denn stets kann man sie, analog den beiden oben erwähnten Beispielen Geomorphologie und Stadtgeographie, als Aussagensysteme interpretieren, deren AV bestimmte Klassen chorologisch-geosphärischer Sachverhalte beinhalten und mithin Teilmengen der Gesamtmenge der geographischen AV bilden.

Keineswegs evident ist dies dagegen hinsichtlich zahlreicher weiterer, meist jüngerer «Geographien» (mit meist korrespondierenden "Arbeitskreisen») wie etwa Zeitgeographie, Verhaltensgeographie, Musikgeographie, Religionsgeographie, feministische Geographie, Geomedizin/Medizinische Geographie, Wahrnehmungsgeographie usw.

Soweit hierbei je bestimmte, chorologisch-geosphärisch relevante Klassen von Einfluß-, also Erklärungsfaktoren untersucht werden (zum Beispiel Zeit, Religion), konstituieren die betreffenden Gebiete definitionsgemäß keine Teildisziplinen der Geographie. Vielmehr haben sie den Status von «Geofaktorenlehren» im eigentlichen 
Sinn. Zwar ist der Terminus «Geofaktor» bzw. «Geofaktorenlehre» bereits als Gattungsbegriff zur Charakterisierung allgemeingeographischer Teildisziplinen vergeben. Da diese Verwendung jedoch offenkundig falsch ist (es handelt sich bei den Gegenstandsbereichen allgemeingeographischer Teildisziplinen eben nicht um Geofaktoren, also um verursachende / erklärende Kräfte, sondern um verursachte / zu erklärende Sachverhaltsklassen), sollte man sie kritisch überprüfen und den Terminus "Geofaktor» bzw. "Geofaktorenlehre» nur zur Bezeichnung geochorologisch relevanter UV bzw. UV-Klassen benutzen. Als «Geographien» dürfte man solche und methodologisch vergleichbare Arbeitsgebiete jedoch nur dann bezeichnen, wenn und soweit die in ihnen untersuchten Sachverhalte paradigmatisch konsistent auch als AV fungieren (können).

Soweit die untersuchten Gegenstandsbereiche dagegen weder geochorologische AV noch geochorologisch relevante UV darstellen, handelt es sich folglich weder um geographische Teildisziplinen noch um Geofaktorenlehren (wie zum Beispiel im Falle der Musikgeographie). In solchen Fällen bewegt man sich offenkundig außerhalb der Geographie, so daß der Terminus «Geographie» hier keine Verwendung finden darf.

Wie man sieht, kann mit Hilfe des AV/UV-Konzeptes auch die innerdisziplinär und hier geographieintern so grundlegende disziplinsystematische Frage geklärt sowie zwischen geographischen Teildisziplinen als den AV-gemäßen systematischen Untergliederungen der Geographie und Geofaktorenlehren als den UV-gemäßen Theoriesystemen unterschieden werden.

Auch hinsichtlich der nachbarwissenschaftlichen Verflechtungen geographischer Teildisziplinen kann das AV/UV-Konzept, analog zum interdisziplinären Verflechtungsbereich, nützlich sein. Diese Verflechtungen sind oft so eng, daß eine Unterscheidung zwischen geographischen Teildisziplinen und korrespondierenden Nachbardisziplinen schwierig ist. Um so schwieriger ist dies, als Nachbarwissenschaften der Geographie ja gleichfalls mit Gegenstandsbereichen der Geosphäre befaßt sind. Besonders offenkundige Fälle dieser Art sind beispielsweise Bodengeographie-Pedologie, Klimageographie-Meteorologie, Stadtgeographie-Stadtplanung, usw. Erst die Isolierung und Bestimmung der AV läßt den Unterschied zwischen den jeweiligen Paaren von Disziplinen erkennen. Erst wenn beide AV-Komponenten, Realobjekt und Erkenntnisobjekt bzw. Gegenstand und Fragestellung, identifiziert sind, werden die disziplinären Zugehörigkeiten erkennbar. So sind zwar die Gegenstände der geographischen Nachbarwissenschaften in der Regel klassische Bestandteile der Geosphäre (wozu hier, im Sinne von CAROL 1956; 1963, auch die Anthroposphäre gerechnet wird). Die Fragestellung, die zweite AV-Komponente, verfolgt jedoch eine andere Intention.

So fragt von den oben genannten Beispielen die Pedologie nach den chemisch-physikalisch-biologischen Eigenschaften des Bodens, die Bodengeographie dagegen nach der räumlichen Ordnung der verschiedensten Bo- denbestandteile und -eigenschaften. Analoges gilt für Meteorologie und Klimageographie in bezug auf die Atmosphäre. Die Stadtplanung dagegen will nicht, wie die Stadtgeographie, vorgefundene stadträumliche Ordnung analytisch erfassen und erklären, sondern stadträumliche Ordnung überhaupt erst schaffen oder verändern. Insofern stellt die Stadtplanung selbst eine UV dar und schafft AV, kehrt also die klassische erkenntnisorientierte in eine technologisch orientierte Variablenbeziehung um. Indem sie somit, wenngleich wissenschaftlich fundiert und gestützt, stadträumliche Realität schafft, ist sie eine angewandte oder technologische und keine erkenntnisorientierte Disziplin. In analoger Weise kann für jede weitere nachbarwissenschaftliche Relation verfahren und der methodologische Unterschied identifiziert werden, wenngleich dieser in der wissenschaftlichen Praxis eher nachgeordnet ist.

\subsection{Allgemeine und Regionale Geographie}

Einen weiteren Anwendungsbereich des AV/UV-Konzeptes stellt die Unterscheidung zwischen Allgemeiner und Regionaler Geographie dar. Zwar handelt es sich auch hierbei um einen Fall innerdisziplinärer Systematisierung, der insofern auch in Kapitel 4.2 hätte behandelt werden können. Doch geht es hierbei ja nicht, wie im Kapitel 4.2, um klassenlogisch definierte, also objekttheoretische innergeographische Gegenstandsbereiche unterschiedlichen Hierarchisierungsniveaus, sondern um erkenntnistheoretische, also metatheoretische Ansätze und Prinzipien geographischer Weltbetrachtung. Insofern ist es zweckmäßig, wenn nicht gar notwendig, diese Frage abgetrennt von der innergeographischen Systematisierung des Kapitels 4.2 zu behandeln. Um Allgemeine von Regionaler Geographie unterscheiden zu können, ist zunächst eine Vorklärung zu treffen. Diese betrifft den Generalisierungsgrad von Aussagen. Folgt man auch in dieser Hinsicht WEINGARTNER $\left({ }^{2} 1978\right.$, S. 32-36) und ergänzend CHALMERS (1986, S. 2-4) sowie ZECHA (1978, S. 58-66), so ist zwischen singulären, partikulären und universalen Aussagen zu unterscheiden (vgl. auch KÖCK 1991, S. 46):

- Eine Aussage ist singulär, wenn sie sich auf ein bestimmtes Element oder mehrere bestimmte, einzeln aufgeführte Elemente einer Klasse von Elementen bezieht und dieses bzw. diese entsprechend mit Singulärbzw. Individualbegriffen benennt, wie z. B.: Deutschland hat eine Hauptstadt.

- Eine Aussage ist partikulär, wenn sie sich auf eine bestimmte Teilgesamtheit einer Klasse von Elementen bezieht und diese mit einem Partikulärbegriff (also Verknüpfung mindestens eines Individualbegriffs mit einem oder mehreren Allgemeinbegriffen) benennt, wie z. B.: Alle Staaten Europas haben Hauptstädte.

- Eine Aussage ist universal, wenn sie sich auf alle Elemente einer Klasse von Elementen bezieht und diese mit einem Universal- bzw. Allgemeinbegriff benennt, wie z. B.: Alle Staaten haben Hauptstädte. 
Vor dem Hintergrund dieser Vorklärung läßt sich nun definieren:

- Allgemeine Geographie ist dasjenige chorologischgeosphärische Aussagensystem, dessen AV universale Aussagen sind. Umgekehrt: eine chorologisch-geosphärische Aussage ist dann allgemeingeographisch, wenn sie eine universale $\mathrm{AV}$ ist.

- Regionale Geographie ist dann dasjenige chorologisch-geosphärische Aussagensystem, dessen AV singuläre oder partikuläre Aussagen sind. Umgekehrt: eine chorologisch-geosphärische Aussage ist dann regionalgeographisch, wenn sie eine singuläre oder partikuläre $\mathrm{AV}$ ist. $\mathrm{Da}$ die Regionale Geographie singuläre und partikuläre AV einschließt, ist folgerichtig; denn in beiden Fällen handelt es sich um raumzeitlich eindeutig bestimmbare bzw. bestimmte, wenngleich unterschiedlich zahlreiche Elemente von Klassen.

Nun stellt sich natürlich die Frage, weshalb man auch in diesem Zusammenhang, also um Allgemeine Geographie und Regionale Geographie bzw. allgemeingeographische und regionalgeographische Aussagen unterscheiden bzw. definieren zu können, der AV bedarf. War es in Kapitel 4.1 die transdisziplinäre Herkunft der UV, die, neben anderen Argumenten, einsichtig machte, weshalb es bei der Definition und Abgrenzung einer Disziplin auf die AV ankommt, so ist es in diesem Zusammenhang die logische Struktur der Erklärung, des UV-Systems also, die den Ausschlag gibt. Folgt man nämlich, wie es hier geschieht, der Erklärungsstruktur entsprechend dem HO- bzw. DN-Schema nach HEMPEL/ OPPENHEIM (1948: $\mathrm{H}$ und $\mathrm{O}$ stehen für Hempel und Oppenheim; D und $\mathrm{N}$ stehen für deduktiv und nomologisch), so benötigt man zur Erklärung allgemeingeographischer Aussagen zwar nur allgemeine Aussagen, wenngleich unterschiedlichen Generalisierungsgrades; zur Erklärung regionalgeographischer Aussagen benötigt man jedoch sowohl allgemeingeographische (universale) als auch regionalgeographische (singuläre oder partikuläre) Aussagen. Die beiden folgenden Beispiele mögen dies verdeutlichen:

- Erklärung der allgemeingeographischen Aussage «Die Begradigung eines Flusses führt zur Erhöhung seiner Fließgeschwindigkeit.»:

- Explanans:

Allgemeines Gesetz: Wenn sich das Gefälle eines Flusses erhöht, erhöht sich seine Fließgeschwindigkeit.

Spezielles Gesetz: Wenn man einen Fluß begradigt, erhöht sich sein Gefälle.

- Explanandum:

Wenn man einen Fluß begradigt, erhöht sich seine Fließgeschwindigkeit.

- Erklärung der regionalgeographischen Aussage «Seit seiner Begradigung im 19. Jahrhundert hat der Rhein eine höhere Fließgeschwindigkeit.»:

- Explanans:

Allgemeines Gesetz: Wenn man einen Fluß begra- digt, erhöht sich seine Fließgeschwindigkeit.

Randbedingung: Im 19. Jahrhundert wurde der Rhein begradigt.

- Explanandum:

Seit seiner Begradigung im 19. Jahrhundert hat der Rhein eine höhere Fließgeschwindigkeit.

Weil somit, wie in diesen Beispielen, dieselbe allgemeingeographische Aussage, hier also "Wenn man einen Fluß begradigt, erhöht sich seine Fließgeschwindigkeit», in zwei methodologisch unterschiedlichen Aussagesystemen (einmal: allgemeingeographisch; das andere Mal: regionalgeographisch) und Rollen (einmal: $\mathrm{AV}$; das andere Mal: UV) vorkommen kann, ist die Identifikation des jeweiligen Aussagensystems als allgemeingeographisch oder regionalgeographisch wiederum nur auf der Grundlage der AV möglich. Nur auf dieser Ebene ist zu erkennen, welcher Sachverhalt letztlicher Gegenstand des Erkenntnisprozesses ist und ob es sich bei ihm eben um einen allgemeingeographischen oder um einen regionalgeographischen Sachverhalt handelt.

\subsection{Wissenschaftssystematische Klassifizierung und Typisierung}

Als viertes und letztes Anwendungsgebiet des AV/UVKonzeptes soll nun noch die methodologische Klassifizierung bzw. Typisierung der Geographie betrachtet werden. Dabei bieten sich wiederum zwei Typisierungsarten an, und zwar die objekttheoretische einerseits, die metatheoretische andererseits.

$\mathrm{Da} ß$ auch die objekttheoretische Klassifizierung der Geographie wiederum nur unter Bezug auf die AV eindeutig möglich ist, ergibt sich aus denselben Gründen, wie sie bereits für die Definition und Abgrenzung bzw. für die innere Untergliederung der Geographie genannt wurden. Aus dem Umstand, daß die AV der Geographie chorologisch-geosphärische Sachverhalte beinhalten, folgt, da $\beta$ die Geographie im begrifflich weiteren Sinne eine Geowissenschaft, im begrifflich engeren Sinne eine spezialisierte, nämlich eine chorologische Geowissenschaft ist. Da ihre AV aber chorologische = raumlogische Sachverhalte zum Gegenstand haben und der Raum das erkenntnisleitende Paradigma der Geographie bildet, ist die Geographie zugleich eine Raumwissenschaft (vgl. auch 4.1). Weitergehende objekttheoretische Typisierungen der Geographie gelten nur noch für je einen ihrer beiden Hauptbereiche. So ist die Geographie mit dem einen ihrer beiden Hauptbereiche, der Physischen Geographie, Naturwissenschaft, da ihre diesbezüglichen AV natürlichen Ursprungs sind. Mit dem anderen Hauptbereich, der Anthropogeographie, ist sie dann Kulturwissenschaft, da ihre diesbezüglichen AV kulturellen Ursprungs sind. Auf die gleichwohl nicht verkennbare Problematik, weil Mißverständlichkeit der letzteren Typisierung soll hier nicht näher eingegangen, sondern nur hingewiesen werden, da es ja nur um die Demonstration der diesbezüglichen Eignung der AV 
geht. Da die anthropogenen AV der Geographie letztlich immer auch gesellschaftlich bzw. sozial bedingt sind, ist die Anthropogeographie außer Kulturwissenschaft zugleich auch Sozial- bzw. Gesellschaftswissenschaft, wobei auch hier auf die mögliche Mißverständlichkeit beider Begriffe im geographischen Kontext hingewiesen sei.

$\mathrm{Da}$ WEINGARTNERS Kriterium erst recht für die metatheoretische Typisierung der Geographie gilt, ist angesichts des in Kapitel 2 Gesagten evident. In diesem Sinne ist die Geographie zunächst eine Real- bzw. empirische Wissenschaft, da und insofern ihre AV empirische Sachverhalte (= Realobjekt) zum Gegenstand haben. Zugleich ist die Geographie jedoch auch Formalwissenschaft, da und insofern ihre AV qua Erkenntnisobjekt «Raum» ein formales Objekt bzw. Konstrukt zum Gegenstand haben. Nicht hingegen ist die Geographie eine normative, technologische oder prognostische Wissenschaft, auch wenn sie unter Modifikation des DN-Schemas (vgl. Kapitel 4.3) gelegentlich normative, technologische oder prognostische Aussagen formuliert. Denn diese haben nicht, wie die Geographie, empirisch gegebene, sondern erst zukünftig sein-sollende, angestrebte oder zu erwartende Erdsachverhalte zum Gegenstand. Allenfalls als «Angewandte Geographie» wäre die Geographie durch Prädikate wie normativ, technologisch oder prognostisch zu charakterisieren.

\section{Schlußbemerkung}

Und was hat man nun von alledem? Gibt es in heutiger Zeit nicht Wichtigeres zu erforschen als ausgerechnet methodologische Fragen, Fragen aus dem Elfenbeinturm also, zumal solche, die in der konkreten Forschungspraxis allenfalls eine randliche Rolle spielen? Gewiß gibt es das! Und dennoch sollte eine methodologische Standortbestimmung und Selbstvergewisserung von Zeit zu Zeit erlaubt sein. Ihre Beherzigung in der konkreten Forschungspraxis hilft nicht nur, die «richtigen» Fragen zu stellen und die «richtigen» Antworten zu suchen, sondern auch, der Außenwelt ein schärferes Profil von dem, was Geographen tun, zu vermitteln. Nicht ohne Grund werden diesbezüglich ja immer wieder kritische Fragen gestellt! Angesichts des steigenden Rechtfertigungsdrucks, um nicht zu sagen «Exhibitionismuszwangs» ist die Gefahr, "Alleswoller» (DÜRR 1986) zu sein, besonders groß. Ob das am Ende nicht gar kontraproduktiv ist? Eine stringenter methodologischer roter Faden täte da sicher gut!

\section{Literatur}

ALBERT, H. (1987): Kritik der reinen Erkenntnislehre. Das Erkenntnisproblem in realistischer Perspektive. Tübingen.

ATTESLANDER, P. ( $\left.{ }^{7} 1993\right):$ Methoden der empirischen Sozialforschung. Berlin.

BAHRENBERG, G. (1987.1): Raum und Geographie. Einleitung. In: BAHRENBERG, G., et al. (Hrsg. 1987): Geographie des Menschen. Dietrich Bartels zum Gedenken. Bremen, S.141-145.

BAHRENBERG, G. (1987.2): Über die Unmöglichkeit von Geographie als "Raumwissenschaft" - Gemeinsamkeiten in der Konstituierung von Geographie bei A. HETTNER und D. BARTELS. In: BAHRENBERG, G., et al. (Hrsg. 1987): Geographie des Menschen. Dietrich Bartels zum Gedenken. Bremen, S. 225-239.

BOBEK, H. (1957): Gedanken über das logische System der Geographie. In: Mitteilungen der Geographischen Gesellschaft Wien, S. 122-145.

CAROL, H. (1956): Zur Diskussion um Landschaft und Geographie. In: Geographica Helvetica, S. 111-133.

CAROL, H. (1963): Zur Theorie der Geographie. In: Mitteilungen der Österreichischen Geographischen Gesellschaft, Band 105, H."I/II, S. 23-38.

CHALMERS, A.F. (1986): Wege der Wissenschaft. Einführung in die Wissenschaftstheorie. Berlin.

DACEY, M. F. (1979): A framework for examination of theoretic viewpoints in geography. In: GALE, S., and OLSSON, G. (Hrsg. 1979): Philosophy in geography. Dordrecht, S. 39-52.

DÜRR, H. (1979): Für eine offene Geographie, gegen eine Geographie im Elfenbeinturm. Karlsruhe.

DÜRR, H. (1986): Was könnte das sein: eine geographische Theorie? In: KÖCK, H. (Hrsg. 1986): Theoriegeleiteter Geographieunterricht. Lüneburg, S. 197-213.

HEMPEL, C. G., and OPPENHEIM, P. (1948): The logic of explanation. In: FEIGL, H., et al. (Hrsg. 1953): Readings in the philosophy of science. New York, S. 319-352.

KÖCK, H. (1987.1): Chorische Logik - die Grundperspektive geographischer Weltbetrachtung. In: BAHRENBERG, G., et al. (Hrsg. 1987): Geographie des Menschen. Dietrich Bartels zum Gedenken. Bremen, S. 179-194.

KÖCK, H. (1987.2): Räumliche Ordnung - universale und geographische Kategorie. In: KÖCK, H. (Hrsg. 1987): Mensch und Raum - Paul Schäfer zum 65. Geburtstag gewidmet. Hildesheim, S. 31-48.

KÖCK, H. (1991): Didaktik der Geographie: Methodologie. München.

KUTSCHERA, F. v. (1972): Wissenschaftstheorie, Band I. München.

LESER, H. (1980): Geographie. Braunschweig.

MAYNTZ, R., HOLM, K., und HÜBNER, P. (21971): Einführung in die Methoden der empirischen Soziologie. Opladen.

NEEF, E. (1956): Die axiomatischen Grundlagen der Geographie. In: Geographische Berichte, S. 85-91.

NEEF, E. (1963): Topologische und chorologische Arbeitsweisen in der Landschaftsforschung. In: Petermanns Geographische Mitteilungen, S. 249-259.

NEEF, E. (1967): Die theoretischen Grundlagen der Landschaftslehre. Gotha.

UHLIG, H. (1970): Organisationsplan und System der Geographie. In: Geoforum, H. 1, S. 19-52.

WEINGARTNER, P. (21978): Wissenschaftstheorie I. Einführung in die Hauptprobleme. Stuttgart-Bad Cannstatt.

WERLEN, B. (1995): Sozialgeographie alltäglicher Regionalisierungen. Band 1: Zur Ontologie von Gesellschaft und Raum. Stuttgart.

ZECHA, G. (1978): Irr- und Umwege der neueren Didaktik: Die Lösung des "ungelösten Deduktionsproblems in der Curriculumforschung". In: MACHOLD, M., et al. (Hrsg. 1978): Österreichische Beiträge zur Bildungsforschung. Wien, S. 55-69. 